


\section{Pulmonary carcinoid tumours}

\section{Educational aims}

1 To describe the epidemiology and prognosis of pulmonary carcinoid tumours.

1 To discuss the pathophysiology and clinical presentation of pulmonary carcinoid tumours.

1 To outline the diagnostic procedures and treatment strategies in carcinoid metastatic disease.

1 To highlight the importance of carcinoid-related heart disease and carcinoid syndrome in the prognosis of carcinoid tumours.

\section{Summary}

Carcinoid tumours are neuroendocrine tumours derived from enterochromaffin cells, which are widely distributed in the body. Pulmonary carcinoids account for $25 \%$ of all carcinoid tumours and they comprise $1-2 \%$ of all lung tumours. The incidence of carcinoid tumours seems to be sharply increasing.

Carcinoid tumours can be classified as typical or atypical, ranging from low-grade malignant to high-grade malignant, according to histological characteristics and differentiation level. Carcinoids can be placed in a spectrum of neuroendocrine tumours, ranging from low-grade malignant typical carcinoid to intermediate atypical carcinoid, to highgrade large cell neuroendocrine carcinoma and small cell lung carcinoma.

Carcinoid pulmonary tumours can present with lymph node or distant metastases, most frequently at the liver. Carcinoid tumours have the ability to secrete many vasoactive peptides, most frequently serotonin. The hormonal secretion is related to systemic manifestations of carcinoid syndrome and carcinoid heart disease. Combining new diagnostic and treatment modalities in metastatic carcinoid patients may result in better prognosis.

The review aims to elucidate the reader on the many aspects of carcinoid pulmonary tumours.

Carcinoids were first described in 1888 by Lubarsch who found multiple tumours in the distal ileum of two patients at autopsy. The term "karzinoide" was first used in 1907 by Oberndorfer to describe similar tumours that were morphologically distinct and with a more indolent behaviour than classical adenocarcinomas $[1,2]$.

Carcinoid tumours are neuroendocrine tumours derived from enterochromaffin cells, which are widely distributed in the body. For this reason, they may be found at any location in the body, but most commonly involve the lungs and gastrointestinal tract $[1,2]$. The term neuroendocrine was introduced based on the capacity of these cells to produce hormonal products that are identical to cell products of the nervous system [3]. They are potentially malignant depending on the aggressiveness of the growth, site of the origin, size of the tumour and the presence of distant metastases [4].
This review is published in conjunction with the case presentation on page 367.

S. André

J.M. Correia

M. Raposo

C. Matos

F. Nogueira

M.C. Abreu

Pulmonary Oncology Unit, Dept of Pulmonology, Egas Moniz Hospital, Lisbon, Portugal.

Correspondence:

S. André

Dept of Pulmonology

Egas Moniz Hospital, SA

Rua da Junqueira, 126

Lisboa

Portugal

E-mail: sandra.andre@sapo.pt

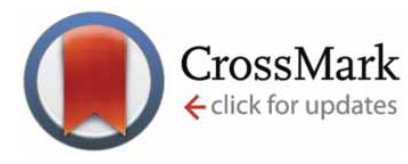

The ERS designates this educational activity for a maximum of 1 CME credit. For information on how to earn CME credits, see page 380. 


\section{Glossary}

Carcinoid syndrome

A paraneoplastic syndrome caused by systemic release of high levels of tumour vasoactive substances from a carcinoid tumour, of which serotonin is the most frequent. Its characteristic symptoms are diarrhoea, flushing and wheezing. It is a potentially life-threatening complication, and treatment should be focused on supportive care and reducing the hormonal peptides excreted from the carcinoid tumour.

Metaiodobenzylguanidine (MIBG)

An analogue of an amine precursor, taken up by chromaffin cells and stored in neurosecretory granules of carcinoid tumour cells. Its labelled form is also used for the diagnosis and treatment of carcinoid tumours.

ISomatostatin analogues

These have a high affinity for somatostatin receptors, especially subtypes 2 and 5 , which are expressed on the cell membranes of carcinoid tumours. Octreotide, a long-acting somatostatin analogue, has been widely used for both the detection and treatment of carcinoid tumours.

5-hydroxyindoleacetic acid (5-HIAA)

Breakdown product of serotonin that is excreted in urine. It is an important biomarker of serotonin production, and has been crucial for the diagnosis and follow-up of carcinoid tumour.

I Cronogranin $\mathrm{A}(\mathrm{CgA})$

Released into the circulation in most neuroendocrine tumours, it is a general, useful, but non-specific tumour marker for even non-functioning tumours. It is important in the follow-up of carcinoid patients and has a high accuracy in the detection of relapse.

Histologically, carcinoid tumours are characterised by positive reactions to silver stains, and to markers of neuroendocrine tissue, such as neuron-specific enolase, synaptophysin and cromogranin. On electron microscopy, they reveal numerous membrane-bound neurosecretory granules that are responsible for the synthesis, storage and release of vasoactive peptides, of which serotonin is the most common $[1,2]$.

The release of tumour vasoactive substances into the systemic circulation is responsible for carcinoid syndrome and probably carcinoid-related heart disease. The clinical outlook depends on the profile of the released products [1-3].

\section{Classification}

Carcinoid tumours have traditionally been classified according to their embryological origin. Foregut carcinoid tumours mostly localise in the thymus, lung, pancreatic islets, stomach and duodenum; midgut carcinoid tumours in the small intestine, appendix and proximal large bowel; and hindgut carcinoid tumours in the distal colon and rectum $[1,2]$.

Carcinoid tumours have actually been classified into typical and atypical carcinoids according to histopathological criteria (cellular morphology, mitotic index and necrosis), which have also proved useful in predicting prognosis (table 1). They can be placed in a spectrum of neuroendocrine tumours, ranging from lowgrade malignant typical carcinoid to intermediate atypical carcinoid, to high-grade large cell neuroendocrine carcinoma and small cell lung carcinoma. Rarely, combined tumours of carcinoid and adenocarcinomas have been reported $[3,5]$. Survival for an atypical carcinoid is significantly worse than that for a typical carcinoid and significantly better than that for large cell neuroendocrine carcinoma and small cell lung carcinoma [6].

\section{Table 1 Classification of carcinoid pulmonary tumours}

\begin{tabular}{|c|c|c|c|}
\hline Type & $\begin{array}{l}\text { Presumed } \\
\text { cell of origin }\end{array}$ & $\begin{array}{l}\text { Histological } \\
\text { features }\end{array}$ & $\begin{array}{c}\text { Clinical } \\
\text { characteristics }\end{array}$ \\
\hline Typical carcinoid & $\begin{array}{c}\text { Epithelial } \\
\text { endocrine cell }\end{array}$ & $\begin{array}{l}\text { No necrosis, }<2 \\
\text { mitosis } / 2 \mathrm{~mm}^{2}\end{array}$ & $\begin{array}{l}\text { Usually indolent, may } \\
\text { secrete corticotropin, } \\
\text { rarely secretes serotonin }\end{array}$ \\
\hline Atypical carcinoid & $\begin{array}{c}\text { Epithelial } \\
\text { endocrine cell }\end{array}$ & $\begin{array}{l}\text { Focal areas of } \\
\text { necrosis, } 2-10 \\
\text { mitosis } / 2 \mathrm{~mm}^{2}\end{array}$ & $\begin{array}{l}\text { Usually aggressive, with } \\
\text { incidence of metastases }\end{array}$ \\
\hline
\end{tabular}


Typical pulmonary carcinoids are usually indolent, with metastases reported in $<15 \%$ of cases and a 5-year survival rate of $>90 \%$. Atypical pulmonary carcinoids comprise approximately one third of all pulmonary carcinoids. They have a more aggressive clinical course with a higher rate of metastatic disease and a worse prognosis (5-year survival ranging 40-60\%) [1]. Approximately $80 \%$ of pulmonary carcinoids are located centrally at the hilus of the lung, the rest being peripheral $[3,4]$.

Common sites of carcinoid tumour metastases are the liver, bone, brain, adrenal glands and ovary. More rarely, metastases in the breast, abdominal wall, spleen, pancreas, pituitary, skin and retro-orbital region have been reported [3].

\section{Epidemiology}

The estimated annual incidence of carcinoid tumours in the USA is 1-2 cases per 100,000 people. However, considering the high number of incidental carcinoid tumours found at autopsy, incidence can actually be much higher [1]. In fact, the overall incidence of carcinoid tumours seems to have sharply increased over the past few decades. It is unknown if this corresponds to a true increase or to a more accurate diagnosis supported by new diagnostic techniques $[2,3]$.

The sex distribution of overall incidence of carcinoid tumours is almost equal. However, in those aged $<50$ years, the carcinoid incidence in females is almost twice that in males [3]. With regards to age-specific incidence rates, peaks at 15-25 years and 65-75 years can be found. The mean age of carcinoid diagnosis is $\sim 47$ years. Atypical carcinoid tumours occur in significantly older patients, most commonly in the sixth decade of life $[2,3]$.

The sites most affected by carcinoid tumours are the gastrointestinal tract and bronchopulmonary tract in 74 and $25 \%$, respectively (table 2) [4]. In $\sim 10 \%$ of cases, the primary tumour site remains unknown. Presentation with distant metastases occurs in $22 \%$ of cases [2].

The pulmonary carcinoids comprise $1-2 \%$ of all lung tumours. The most frequent location is the main or lobar bronchi ( $70 \%$ of cases), with approximately one third of the tumours being located in the periphery of the lungs (mostly atypical carcinoid tumours). In 10\% of cases, regional lymph node involvement is found at the time of diagnosis [3]. Most carcinoid tumours are assumed to be sporadic $[2,4]$.

Although scarce information is known about

\section{Table 2 Tumour location and associated frequency of carcinoid tumours}

$\begin{array}{lc}\text { Location } & \text { Frequency \% } \\ \text { Jejunum, ileum } & 26 \\ \text { Tracheobronchopulmonary } & 25 \\ \text { Appendix } & 19 \\ \text { Rectum } & 13 \\ \text { Colon } & 8 \\ \text { Stomach } & 3 \\ \text { Pancreatic islet } & 3 \\ \text { Duodenum } & 2 \\ \text { Others\# } & 1\end{array}$

Data taken from [4]. \#: oesophagus, ovary, testis, prostate, kidney, breast, skin, etc.

carcinoid tumour risk factors, smoking appears to be directly implicated [3]. In a minority of patients $(<1 \%)$, familial pulmonary carcinoids are found. Familial carcinoids are associated with the multiple endocrine neoplasia type 1 (MEN-1) gene, which is situated on chromosome 11 (11q13). MEN-1 is a familial tumour syndrome, with an autosomal-dominant inheritance, characterised by a high frequency of endocrine malignancies in the parathyroid glands, pancreas, anterior pituitary, lungs, thymus and stomach. Carcinoids in MEN-1 are mostly of foregut origin (thymus, lung, pancreatic islets, stomach and duodenum), whereas midgut and hindgut carcinoids usually do not occur in these patients $[2,4]$. There are rare familial pulmonary carcinoids that are not associated with MEN-1 syndrome and are probably related to occult mutations. In fact, there is a higher risk of developing a carcinoid tumour among firstdegree relatives of patients with a carcinoid tumour. In sporadic midgut carcinoids, a high frequency of deletions on chromosome 18 has been previously described $[2,4]$. Up to $40 \%$ of sporadic lung carcinoids display MEN-1 11q13 loss of heterozygosity and mutation of allele, and they are atypical rather than typical [7].

\section{Pathophysiology}

Carcinoid tumours have the ability to secrete vasoactive peptides, which are initially responsible for clinical presentation and complications. Carcinoids have been found to secrete serotonin (5-hydroxytryptamine (5-HT)), bradykinins, tachykinins, histamine, substance $P$, adrenocorticotropic hormone, prostaglandins, kallikrein, dopamine and several other peptides, although $5-\mathrm{HT}$ production is the most prominent $[1,4]$. 


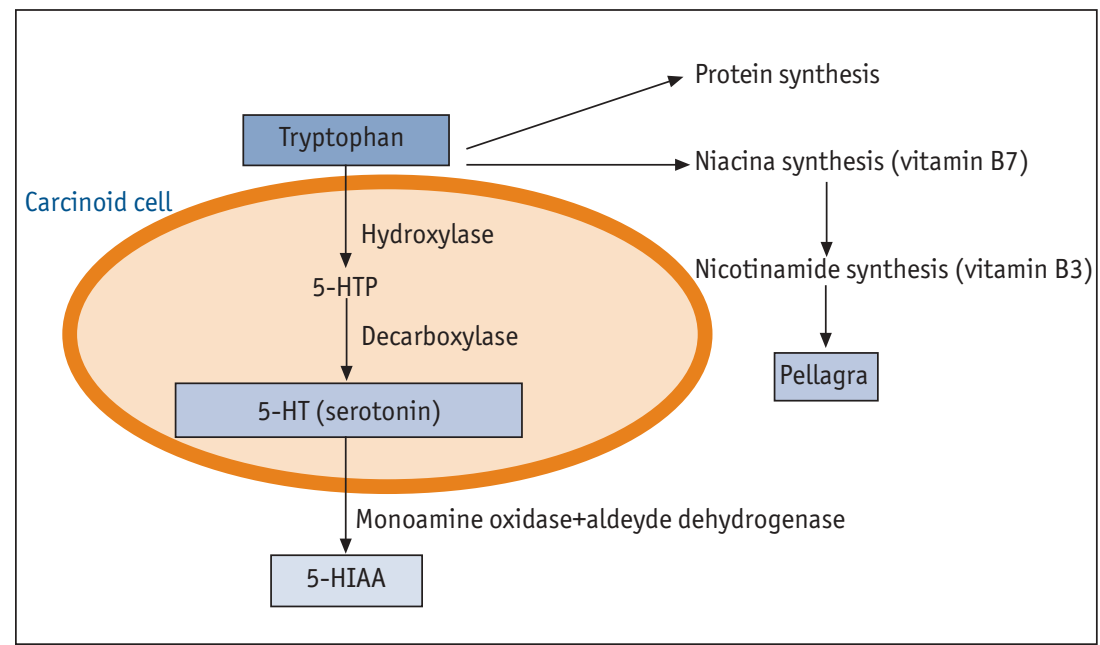

Figure 1

Serotonin metabolism. 5-HTP: 5-hydroxytryptophan.

Under normal conditions, 99\% of dietary tryptophan is metabolised to nicotinamide, and $<1 \%$ is converted into serotonin. In carcinoid tumours, a disequilibrium of tryptophan metabolism results in an overproduction of 5-HT (figure 1).

The breakdown product of $5-\mathrm{HT}$, 5-HIAA, is excreted in the urine and is an important biomarker for carcinoid tumours, as an indicator of $5-\mathrm{HT}$ production in the body. When this shift in tryptophan metabolism is enhanced, which frequently occurs in the presence of extensive metastatic disease, a reduction in nicotinamide production is responsible for a diagnosis of pellagra (vitamin B3 deficiency) $[2,4,8]$.

The release of 5-HT into the systemic circulation is responsible for excitation of smooth muscle, leading to increased gastrointestinal motility or bronchoconstriction, platelet aggregation and vascular constriction (direct and via sympathetic innervation), and dilatation (endothelium dependent). The systemic actions of 5-HT and other vasoactive substances released by carcinoid tumours are thought to cause carcinoid syndrome and carcinoid-related heart disease $[1,2]$.

\section{Clinical features}

In patients with a pulmonary carcinoid tumour diagnosis, local symptoms have been reported in $52-92 \%$ of cases [3]. The most frequent symptoms are haemoptysis, cough, recurrent pulmonary infection, fever, chest discomfort, unilateral wheezing and shortness of breath [2]. The paraneoplastic syndromes are observed when metastasis (mainly liver) has developed.

Systemic signs and symptoms of pulmonary carcinoid tumour are dependent on the expression pattern and release of specific bioactive substances. Rarely, these paraneoplastic syndromes can be the first presentation of a carcinoid. The frequency of metastatic disease related to pulmonary carcinoids is actually very low when compared with gastrointestinal carcinoids $[2,3]$.

\section{Carcinoid syndrome}

The carcinoid syndrome is very rare in patients with pulmonary carcinoids, occurring in $\sim 2 \%$ of cases. Its characteristic symptoms are diarrhoea, flushing, palpitations and, less frequently, wheezing. The severity of these symptoms is related to the location of the tumour (related to drainage to systemic circulation, especially in liver metastases) and tumour mass [2, 3].

$5-\mathrm{HT}$ is the most frequently detected peptide responsible for carcinoid syndrome. In rare cases, carcinoid crisis can be provoked by the release of a large concentration of vasoactive substances into the systemic circulation, after tumour manipulation, induction of anaesthesia or intubation [1-3].

Carcinoid syndrome with severe diarrhoea and flushes can lead to dehydration, hypotension, hydro-electrolytic disturbances and arrhythmias, which are potentially life-threatening complications [2].

\section{Carcinoid heart disease}

Carcinoid heart disease is a late complication that occurs in $20-70 \%$ of patients with metastatic carcinoid tumours with carcinoid syndrome [2]. Carcinoid heart lesions are characterised by fibrosis of right-side valves, caused by exposure to high concentrations of vasoactive substances in this side of the heart. A part of these substances is metabolised in the lung, which explains a significantly lower exposure of the left side of the heart and a lower incidence of left-sided heart disease (10\% of patients) [1].

5-HT plays an important role in the pathogenesis of carcinoid-related heart disease. This is supported by the finding that some anorectic drugs (fenfluramine and dexfenfluramine) and antimigraine drugs (ergotamin and methysergide), which act via the 5-HT pathway, have been associated with valve lesions similar to carcinoid heart disease $[1,2]$. Studies have shown a good correlation between levels of 5-HIAA and carcinoid valvular disease severity [9].

At the end stage, carcinoid heart disease leads to symptomatic right heart failure and secondary pulmonary hypertension, resulting in substantial morbidity and mortality. Valvular 
replacement should be considered in carcinoid heart disease $[1,2]$.

\section{Cushing's syndrome}

Cushing's syndrome is present in $\sim 2 \%$ of patients with carcinoid pulmonary tumour. It is characterised by ectopic adrenocorticotropic hormone production, resulting in hypercortisolism. The typical clinical signs are weakness, hypertension, glucose intolerance, hypokalaemia, alkalosis, weight loss, anaemia and hyperpigmentation $[3,4]$.

\section{Ectopic growth hormone}

Carcinoid tumours are the most frequent causes of ectopic production of growth hormone $(\mathrm{GH})$, leading to high levels of $\mathrm{GH}$ and insulin-like growth factor- 1 with classic acromegaly clinical features $[3,4]$.

\section{Diagnosis}

\section{Pathology}

Diagnosis of pulmonary carcinoid tumour is histological, usually from a bronchial or hepatic metastases biopsy. At bronchoscopy, the tumour typically has a highly vascular appearance, with a pink/reddish or yellow smooth-looking surface. Significant haemorrhage is a frequent complication of bronchoscopy and bronchial biopsy. The biopsy material should be examined by a pathologist who is familiar with carcinoid diagnosis due to frequent problems in differential diagnosis. Depending on the morphology of cells and presence of necrosis and/or mitosis, tumours can be graded as low-grade malignant or high-grade malignant, which is useful for predicting prognosis [1-3].

\section{Radiological findings}

Chest imaging reveals a nodular lesion with a mean size of $\sim 3 \mathrm{~cm}$, most of which are centrally located, with only one-third located peripherally. Computed tomography (CT) scanning can be used for visualising liver metastases and tumour localisation in the mediastinum and lungs. It is also important in the decision-making process for local and metastatic treatment options [2].

\section{Tumour markers}

Laboratory tumour markers are very important in the diagnosis and follow-up of carcinoid tumours. Urinary 5-HIAA, which is a measure of the $5-\mathrm{HT}$ production level, has been considered the gold standard for diagnosis and follow-up of carcinoid patients (if elevated at the time of diagnosis). It has a very high specificity (almost $100 \%$ ) and a much lower sensitivity (35\%). Urinary 5-HIAA levels can be influenced by food (i.e. bananas, avocados, pineapple and walnuts) and medication use [2].

Platelet 5-HT level is a sensitive marker for the detection of small amounts of serotonin. However, its use is limited in cases with a high rate of serotonin excretion, as the platelet serotonin level reaches a maximum and, hence, will be of no use during follow-up.

$\mathrm{CgA}$ is a glycoprotein produced in most neuroendocrine tumours. Its release into the circulation can be used as a non-specific general tumour marker, permitting detection of functioning and non-functioning tumours. The combination of $\mathrm{CgA}$ and urinary 5-HIAA has shown a higher accuracy in the detection of relapse in carcinoid patients [3].

\section{Nuclear scintigraphy}

Immunoscintigraphy by somatostatin analogues is a potentially useful diagnostic study for neuroendocrine lung tumours. This technique is based on the overexpression of somatostatin receptors on carcinoids and the high affinity of octreotide analogues for them. ${ }^{111}$ Indium-pentetreotide (a radioactive-labelled octreotide analogue) scintigraphy has a sensitivity of $80-90 \%$ for the detection of carcinoid tumours. It provides information about the localisation of the tumour (primary and distant disease) and is predictive of response to octreotide therapy $[2,3]$.

${ }^{131}$ Iodine-metaiodobenzylguanidine (131ןMIBG) is an analogue of a biogenic amine

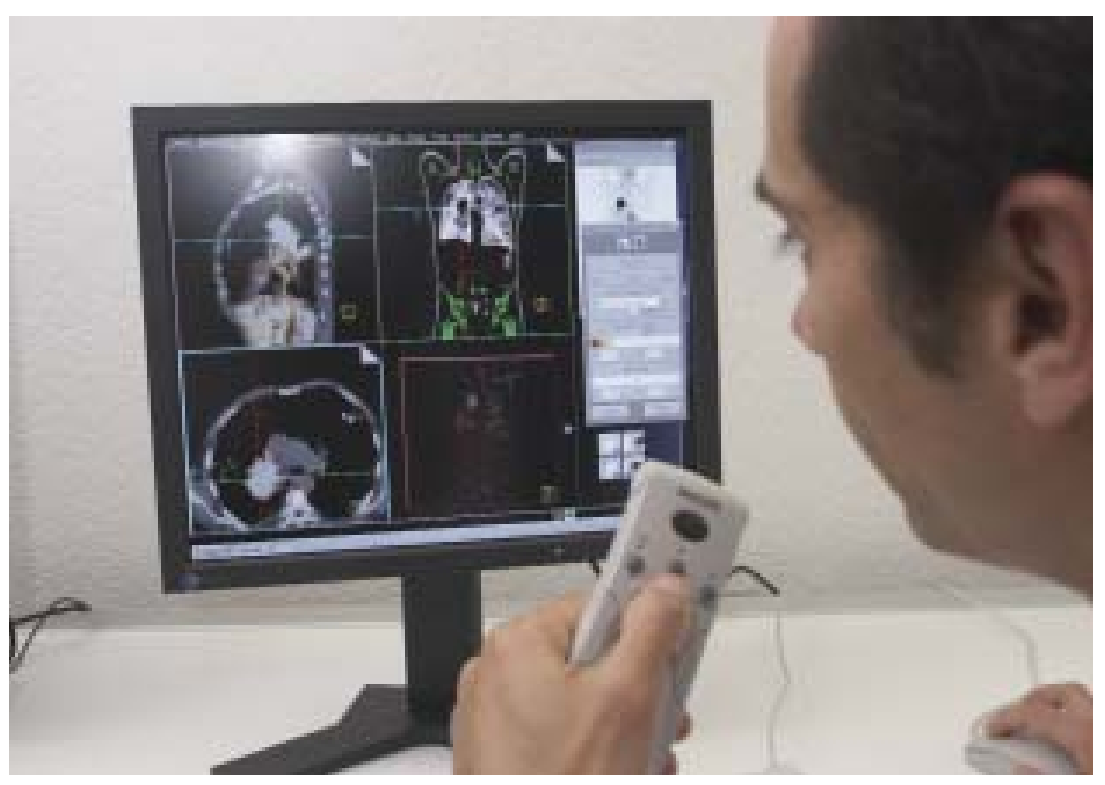


precursor, which is taken up by neuroendocrine cells and stored in neurosecretory granules. ${ }^{131}$ |MIBG is used for the detection and imaging of neuroendocrine tumours and has more recently been used as a therapeutic tool. The sensitivity of the MIBG scan is reported to be lower than the ${ }^{111}$ Indium-pentetreotide scintigraphy scan $(\sim 70 \%)$. However, some studies have shown comparable results between the two scintigraphic techniques. A combination of these scans seems to increase sensitivity to $95 \%$ [2]. Scintigraphic techniques have a low specificity, with positive results for many other tumours, granulomas and autoimmune diseases.

Use of positron emission tomography (PET) with ${ }^{18} \mathrm{~F}$-fluorodeoxyglucose ( ${ }^{18} \mathrm{FDG}$ ) in carcinoid tumours is very limited due to low proliferative activity and high differentiation rate of carcinoids. Some promising results have been described during the follow-up and diagnosis of small lesions and lymph node metastases in PET with tracers directed toward the specific characteristics of carcinoid tumours, 6-[ $\left.{ }^{18} \mathrm{~F}\right]$ fluorodopamine ( ${ }^{18} \mathrm{~F}$-dopa) and ${ }^{11} \mathrm{C}$-labelled 5-HTP $[2,3]$.

Although ${ }^{131}$ I-MIBG and ${ }^{111}$ Indium-pentetreotide scintigraphy has a $70-80 \%$ sensitivity in detecting bone carcinoid metastases, bone scintigraphy shows a higher sensitivity for the detection of these metastases and can be used in patients with clinical suspicion [2].

\section{Cardiac evaluation}

Detection of carcinoid heart disease at an early stage is important in order to optimise therapy and improve prognosis. Patients with carcinoid syndrome should be monitored by echocardiography for the detection of valvular lesions. In some centres, cardiac damage is monitored by brain natriuretic peptide levels, and echocardiography is reserved for patients with cardiac lesion suspicion [2].

\section{Treatment}

\section{Surgery}

Surgery is the treatment of choice for carcinoid pulmonary tumour, offering the only chance of cure. Surgery or endoscopic interventions may be justified, even when metastases have occurred, to avoid local problems [10]. In typical carcinoids, bronchoplastic parenchyma-sparing surgery is the standard surgical procedure in some centres, although systematic mediastinal lymphadenectomy is suggested by other centres. In atypical carcinoids, this is not considered sufficient and lobectomy is the surgical resection procedure most commonly performed [11]. Endobronchial laser treatment has also been advocated as an alternative treatment option in patients who are not fit to undergo surgical resection [3].

\section{Supportive care}

In patients with carcinoid syndrome, quality of life is severely impaired and supportive care is crucial. Flushes can be reduced by avoiding stress and foods known to provoke symptoms (e.g. spicy meals and alcohol). Diarrhoea can be

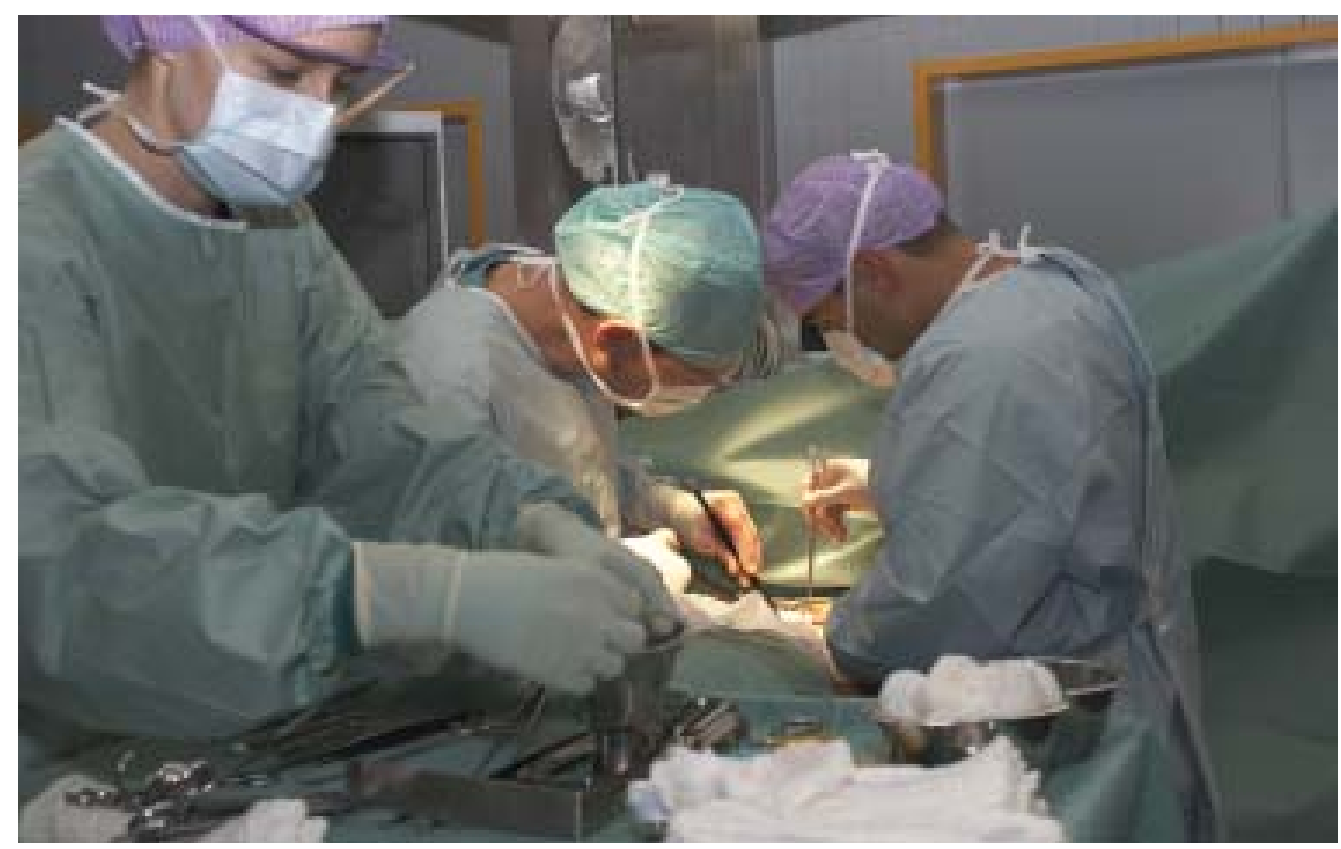


treated with antidiarrhoeal medications (e.g. loperamide and codeine). Supplementation of vitamins and nicotinic acid is recommended to avoid pellagra [2].

\section{Somatostatin analogues}

Somatostatin analogues have a central role in both the diagnosis and treatment of metastatic carcinoid tumours, and they are the first-choice treatment. Somatostatin is a 14-amino-acid peptide that activates somatostatin receptors, inhibiting the secretion of a broad range of hormones. Its short half-life (2-4 minutes) limits its clinical application [1-3].

There are five known somatostatin receptors that have been characterised, which are expressed on $>80 \%$ of carcinoid tumours, especially subtypes 2 and 5 . Octreotide, a somatostatin analogue, has a high affinity for the receptor subtypes 2 and 5, a half-life of 90-120 minutes and can be administered subcutaneously every 6-8 hours. Octreotide can induce symptomatic improvement in up to $80 \%$ of patients, decrease urinary 5 -HIAA excretion in $72 \%$ of patients, and has also been reported to occasionally inhibit tumour growth. Positive ${ }^{111}$ Indium-pentetreotide scintigraphy seems to be predictive for clinical response to treatments with somatostatin analogues $[2,4]$.

\section{Interferon- $\alpha$}

Interferon (IFN)- $\alpha$ was introduced in 1982 as a treatment modality for carcinoid tumours. Although its exact mechanism of action is not yet understood, the currently recognised mechanism involves inhibition of cell proliferation, immune cell-mediated cytotoxicity, control secretion of tumour products and reduction in tumour growth by blocking the cell cycle $[2$, 4].

IFN- $\alpha$ treatment shows decreases in urinary 5 -HIAA in $42 \%$ of patients and reduction of tumour size in only $15 \%$ of cases. In $5-20 \%$ of cases, development of antibodies occurs after a median of 25 months of IFN- $\alpha$ treatment, which is associated with a failure of response to treatment. There are no benefits in increasing the dose of IFN- $\alpha$ or in combining IFN- $\alpha$ and somatostatin analogues [2].

The high number of side-effects, which may include fever, fatigue, anorexia and weight loss, and the lower rate of tumour regression and symptom control result in limited interest in using IFN- $\alpha$ for the treatment of metastatic carcinoid tumours [1].

\section{I-MIBG}

${ }^{131}$ I-MIBG is used in the diagnosis and treatment of pulmonary carcinoid tumours. Its application for carcinoid tumour therapy requires the use of much higher doses of ${ }^{131}$ I-MIBG to provide a selective local effect by internal radiation in the tumour cells in patients with a positive ${ }^{131}$ I-MIBG scan $[2,3]$.

Symptomatic responses have been reported in $60 \%$ of treated patients, but with a short median duration of 8 months. Some authors have reported the use of unlabelled MIBG with similar symptomatic responses, but with a shorter median duration [2].

\section{Radioactive-labelled somato- statin analogues}

Recently, several radioactive-labelled somatostatin analogues have been developed for use in neuroendocrine tumours with positive ${ }^{111}$ Indium-pentetreotide scintigraphy for somatostatin-targeted radiotherapy. ${ }^{111}$ Indiumpentetreotide shows some benefits for the treatment of small tumours or micrometastases with symptomatic improvement in up to $60 \%$ of patients, but effectively reduces the tumour in only a minority of patients. Adverse events can be expected from healthy tissues throughout the body that express somatostatin receptors. Reported side-effects from administering radioactive-labelled somatostatin analogues are nausea, vomiting, and renal and haematological toxicity. The results of large clinical trials are expected in the near future $[2,3]$.

\section{Systemic chemotherapy}

Chemotherapy results are very discouraging in metastatic carcinoid tumours, thus leading to its use only as second-line therapy [4]. The most studied chemotherapy regimens for carcinoid tumours involve streptozocin and cyclophosphamide or streptozocin and 5-fluorouracil, with response rates of $20-30 \%$.

As third-line chemotherapy, a combination of cisplatin and etoposide (a regimen commonly used in the treatment of small cell lung cancer) is used, which has a $67 \%$ response rate in patients with neuroendocrine carcinomas. Patients with typical carcinoid tumours did not respond to this form of therapy [3]. A higher benefit of systemic chemotherapy is observed in more aggressive variants of carcinoid tumour [1].

\section{Radiotherapy}

The use of radiation therapy in the treatment of carcinoid tumours has not been extensively
Educational questions

1. What is the pathophysiology and clinical presentation of carcinoid syndrome?

2. What are the tumour biological markers used for diagnosis and follow-up of carcinoid tumour?

3. What are the clinical manifestations of carcinoid heart disease?

4. What is the first-line treatment for carcinoid metastatic disease? 
studied. It has been shown to be effective in the palliation of bone and central nervous system metastases. Some authors have proposed the use of adjuvant radiotherapy in mediastinal lymph node metastases, but this has not proven beneficial [3].

\section{Management of hepatic metastases}

Surgical resection of liver metastases may be of benefit in patients with limited hepatic disease, resulting in long-term relief of symptoms and prolonged survival. However, liver metastases are usually diffuse at the time of diagnosis, and surgical resection is generally not possible $[1,2]$.

Hepatic artery embolisation is based on the principle that tumours receive circulation from the hepatic artery, whereas hepatocytes can receive blood from the portal venous circulation. The technique consists of infusion of gel-foam powder into the hepatic artery through an angiography catheter. This approach has resulted in improvement of symptoms, a biochemical response rate in $50 \%$ of patients and a reduction in tumour burden. Unfortunately, duration of the response is often short, with a medium duration of 12 months. The side-effects include pain in the liver region, renal toxicity, and, occasionally, hepatorenal syndrome and elevation of liver enzymes with fever [1, 2].

The role of liver transplantation in carcinoid metastatic disease is still unclear. Early series have shown high rates of peri-operative mortality and tumour recurrence, although recent results have been more encouraging, with a $69 \%$ 5-year survival rate in highly selected patients [1].

\section{New therapeutic developments}

Radiofrequency ablation is a new technique that utilises a CT-guided high-frequency electric current to kill tumour cells. It can be used for nodules $\leq 4 \mathrm{~cm}$ in diameter in the treatment of unresectable primary and secondary hepatic tumours. Its use in neuroendocrine tumours has been reported in small series, but shows encouraging results in local tumour control and symptomatic and biochemical response. However, local recurrences and development of new metastases are frequently reported $[2,3]$.

Some trials are been performed using a combination of 5-fluorouracil and IFN- $\alpha$ in the treatment of advanced metastatic carcinoids. The effect of thalidomide in patients with neuroendocrine tumours is also being investigated [3].

\section{Follow-up}

Follow-up should be focused on monitoring tumour size and extension of metastases by CT and nuclear scanning. Special attention should be given to unexpected metastatic patterns, such as bone metastases $[2,3]$.

Hormonal activity must be monitored on a regular basis. Determination of urinary 5-HIAA is very useful in follow-up. CgA is a useful but not specific biochemical marker, with a good correlation with tumour burden. It seems to be an important predictor of an adverse prognosis [1,3].

Routine examinations for carcinoid-related heart disease every 6-12 months are very important in detecting carcinoid heart complications at an early stage in order to adjust therapy and improve prognosis [2].

\section{Prognosis}

Combining new diagnostic and treatment modalities in metastatic carcinoid tumours has resulted in improvements in survival and quality of life. Patients with typical pulmonary carcinoids have a good prognosis, with a 5-year survival of $87-100 \%$ and a 10 -year survival of $82-87 \%$, according to different published data. Patients with pulmonary atypical carcinoids have a worse prognosis: 5 -year survival is $56-75 \%$ and 10 -year survival is $35-56 \%[1-3,12]$.

\section{Conclusions}

Carcinoid pulmonary tumours are relatively frequent but only rarely manifest. The frequency of metastatic disease is significantly lower compared with gastrointestinal carcinoids. The great challenges for carcinoid tumours are the diagnosis and treatment of metastatic disease, where new approaches can result in a better quality of life and longer survival [13].

Due to longer survival times, complications such as carcinoid heart disease and new metastatic patterns, e.g. skin and bone metastases, may become more important features of carcinoid disease. Carcinoid syndrome and carcinoid heart disease are life-threatening complications. Therefore, early detection and treatment oriented towards reducing elevated levels of hormonal excretion are crucial [1,3].

The increasing challenges of diagnosis and therapeutic options for carcinoid disease require a multidisciplinary approach, where a variety of specialists, including the pulmonologist, 
oncologist, surgeon, pathologist, cardiologist, gastroenterologist, radiologist and a nuclear medicine specialist, can discuss therapeutic options for each patient case. For the lowest frequency of carcinoid tumours, reference specialised centres should be created.

\section{References}

1. Kulke MH, Mayer RJ. Carcinoid tumours. N Engl J Med 1999; 340: 858-868.

2. Zuetenhorst JM, Taal BG. Metastatic carcinoid tumours: a clinical review. Oncologist 2005; 10: 123-131.

3. Hage $R$, de la Rivière $A B$, Seldennijk CA, van den Bosch JMM. Update in pulmonary carcinoid tumours: a review article. Ann Surg Oncol 2003; 10: 697-704.

4. Lips CJM, Lentjes EGWM, Höppener JWM. The spectrum of carcinoid tumours and carcinoid syndromes. Ann Clin Biochem 2003; 40: 612-627.

5. Brambilla E, Travis WD, Colby TV, Corrin B, Shimosato Y. The new World Health Organization classification of lung tumours. Eur Respir J 2001; 18: 1059-1068.

6. Debelenko LV, Brambilla E, Agarwal SK, et al. Identification of MEN1 gene mutations in sporadic carcinoid tumors of the lung. Hum Mol Genet 1997; 6: 2285-2290.

7. Travis WD, Rush W, Flieder DB, et al. Survival analysis of 200 pulmonary neuroendocrine tumors with clarification of criteria for atypical carcinoid and its separation from typical carcinoid. Am J Surg Pathol 1998; 22: 934-944.

8. Hegyi J, Schwartz RA, Hegyi V. Pellagra: dermatitis, dementia and diarrhea. Int J Dermatol 2004; 43: 1-5.

9. Møller JE, Connolly HM, Rubin J, Seward JB, Modesto K, Pellikka PA. Factors associated with progression of carcinoid heart disease. N Engl J Med 2003; 348: 1005-1015.

10. Kosmidis PA. Treatment of carcinoid of the lung. Curr Opin Oncol 2004; 16: 146-149.

11. MCMullan DM, Wood DE. Pulmonary carcinoid tumours. Semin Thorac Cardiovasc Surg 2003; 15: 289-300.

12. Thomas CF Jr, Tazelaar HD, Jett JR. Typical and atypical pulmonary carcinoids: outcome in patients presenting with regional lymph node involvement. Chest 2001; 119: 1143-1150.

13. Cerilli LA, Ritter JH, Mills SE, Wick MR. Neuroendocrine neoplasms of the lung. Am J Clin Pathol 2001; 116: Suppl., S65-S95.

\section{Suggested answers}

1. Carcinoid syndrome is a paraneoplastic condition that occurs in $2 \%$ of patients. The release of tumour vasoactive substances in the systemic leads to acute diarrhoea, flushing, palpitations and, less frequently, wheezing. Serotonin is the most frequently detected peptide responsible for carcinoid syndrome. Symptom severity is related to the location of the tumour (drainage to systemic circulation, especially in liver metastases) and tumour mass. Severe carcinoid crisis, leading to dehydration, hypotension and arrhythmias, is a potentially life-threatening complication.

2. Urinary 5-HIAA, which is a measure of the serotonin production level, has been considered the gold standard for diagnosis and follow-up of carcinoid patients. CgA is a glycoprotein produced in most neuroendocrine tumours. It is a non-specific general tumour marker, permitting detection of functioning and non-functioning tumours. The monitoring of $\mathrm{CgA}$ and urinary 5-HIAA is useful for carcinoid tumour follow-up, showing a high accuracy in the detection of relapse.

3. Carcinoid heart disease is characterised by the fibrosis of right-sided valves, caused by exposure to high concentrations of vasoactive substances in this side of the heart. At the end stage, carcinoid heart disease leads to symptomatic right heart failure and secondary pulmonary hypertension, resulting in substantial morbidity and mortality.

4. Treatment should be focused on the reduction of elevated levels of hormonal excretion and supportive care to complications of carcinoid crisis. Surgery is the treatment of choice for pulmonary carcinoid tumour, offering the only chance of cure. Somatostatin analogues have a central role in both the diagnosis and treatment of metastatic carcinoid tumours, and they are the firstchoice treatment for metastatic carcinoid tumours. 